Document downloaded from:

http://hdl.handle.net/10251/102305

This paper must be cited as:

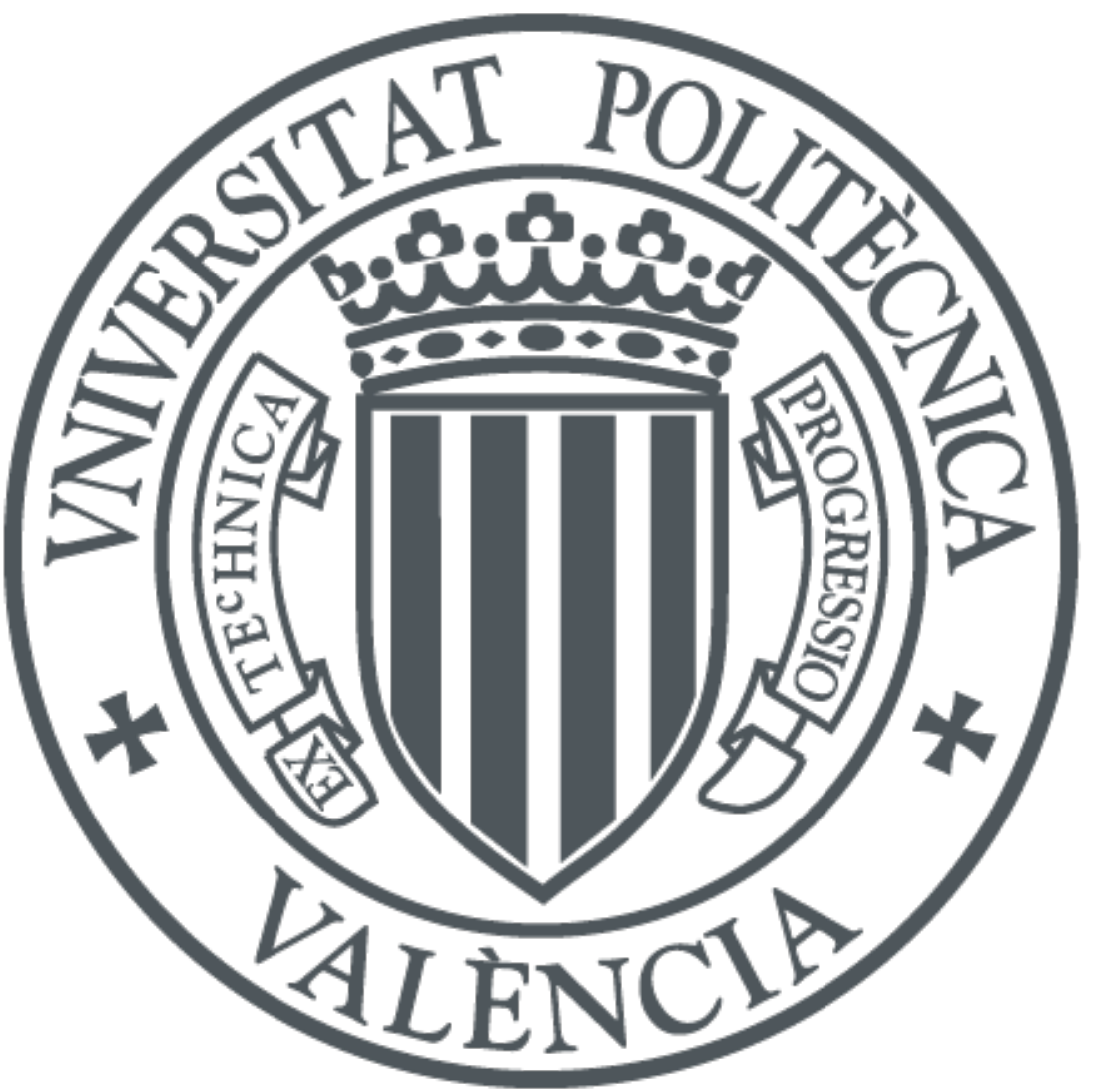

The final publication is available at

https://doi.org/10.1021/acs.bioconjchem.6b00624

Copyright American Chemical Society

Additional Information 


\title{
Improved Performance of DNA Microarray Multiplex Hybridization Using Probes Anchored at Several Points by Thiol-Ene or Thiol-Yne Coupling Chemistry
}

\author{
Maria-Jose Bañuls ${ }^{\mathrm{a}}$, Pilar Jiménez-Meneses ${ }^{\mathrm{a}}$, Albert Meyer ${ }^{\mathrm{b}}$, Jean-Jacques Vasseur ${ }^{\mathrm{b}}$, \\ François Morvan $^{\mathrm{b} *}$, Jorge Escorihuela ${ }^{\mathrm{a}}$, Rosa Puchades ${ }^{\mathrm{a}}$, Ángel Maquieira ${ }^{\mathrm{a} *}$ \\ ${ }^{a}$ Interuniversitary Research Institute for Molecular Recognition and Technological \\ Development (IDM), Chemistry Department, Universitat Politècnica de València, \\ Camino de Vera s/n, 46022 Valencia, Spain
}

\begin{abstract}
*amaquieira@qim.upv.es
${ }^{b}$ Institut des Biomolécules Max Mousseron (IBMM), UMR 5247 CNRS, Université de Montpellier, ENSCM, place Eugène Bataillon, CC1704, 34095 Montpellier Cedex 5, France. *francois.morvan@umontpellier.fr

${ }^{\dagger} J$. Escorihuela present address is Laboratory for Organic Chemistry, Wageningen University, Dreijenplein 8, 6703HB Wageningen, The Netherlands.
\end{abstract}

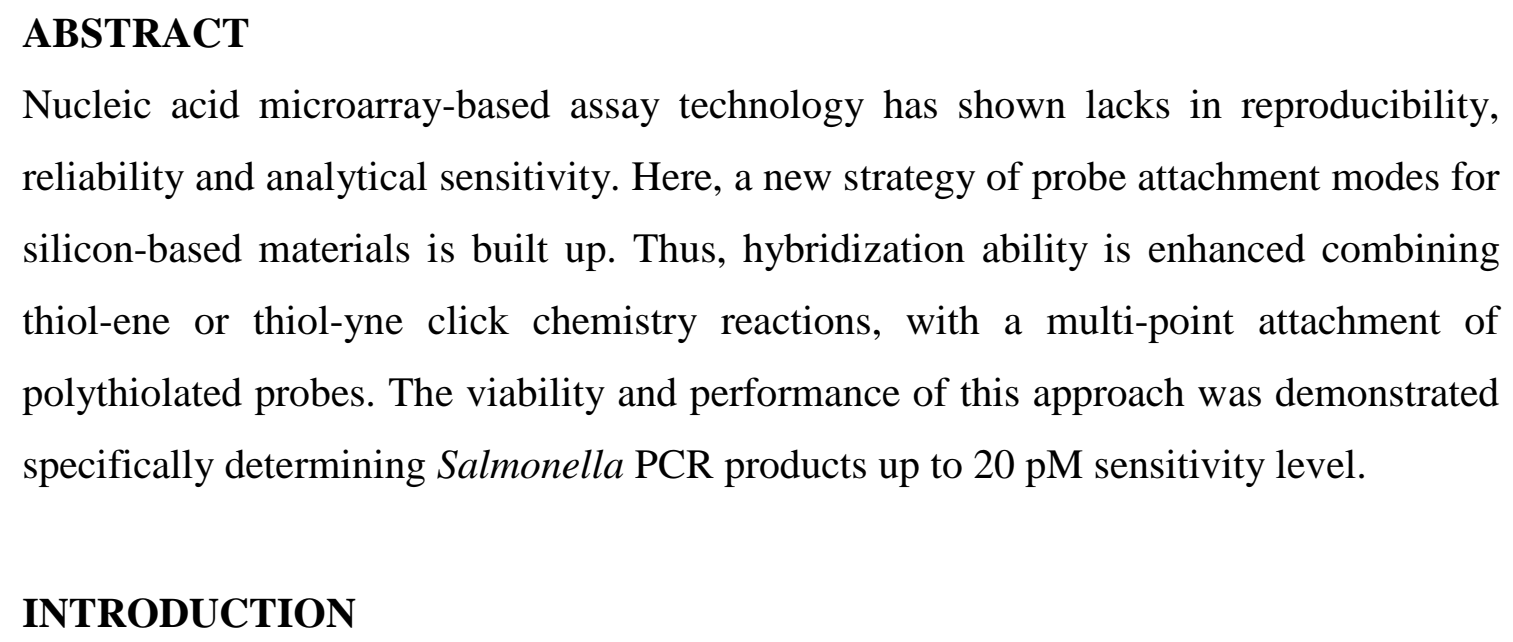
reliability and analytical sensitivity. Here, a new strategy of probe attachment modes for silicon-based materials is built up. Thus, hybridization ability is enhanced combining thiol-ene or thiol-yne click chemistry reactions, with a multi-point attachment of polythiolated probes. The viability and performance of this approach was demonstrated specifically determining Salmonella PCR products up to 20 pM sensitivity level.

INTRODUCTION 
1 The development of high-performance methods for the sensitive and selective detection

2 of DNA and RNA targets has become a key point in biomedical and clinical studies, ${ }^{1}$ 3 agricultural, food and environmental fields. ${ }^{2-4}$ Among the working techniques, 4 microarraying emerges as a tool showing parallel and high throughput assay capabilities. ${ }^{5}$

5 However, both clinical and analytical metrics produced by microarray-based assay 6 technology have recognized lacks in reproducibility, reliability and analytical sensitivity. ${ }^{6}$

7 Most of these drawbacks are attributed to poor probe attachment and solid-liquid interface 8 control. $^{7}$

9 Indeed, the success of microarray-based techniques depends on the good accessibility and functionality of the surface-bound probes, which closely relates to the chemistry of attachment (support nature, probe orientation, probe density, reproducibility). ${ }^{8,9}$ Many work have been developed in this field involving passive immobilization by adsorption forces, ${ }^{10}$ electrostatic interactions, ${ }^{11}$ affinity reactions ${ }^{12}$ and covalent bonding. ${ }^{13-15}$ But, nowadays there is still a need for better attachment modes providing high performance in the developed microarray; specially regarding sensitivity and selectivity. Generally, covalent binding is the preferred approach for the probe attachment, because it provides good stability and high binding strength, controlling also orientation and density of probes. However, it has several drawbacks as the need of linker molecules, slow procedures and crowding effects. ${ }^{7}$

Despite the many methods described for microarray probe covalent anchoring; the most interesting reported approaches to overcome the abovementioned drawbacks, are those based on click chemistry reactions. ${ }^{16}$ Thiol-ene ${ }^{17,18}$ and thiol-yne ${ }^{19,20}$ coupling chemistries belong to this family, which are characterized by orthogonality, high yields, regioselectivity, compatibility with aqueous media, mild reaction conditions, use of benign catalysts and solvents, and high reaction rates. The good performance of these coupling chemistries made them useful for many applications such as in polymers, dendrimers, bioconjugation and surface photografting. ${ }^{21-23}$ However, few examples can be found employing these click reactions for microarray fabrication. ${ }^{24-30}$ Regarding thiol-ene coupling, the most interesting contributions are those by Waldmann and colleagues, ${ }^{27-30}$ but they are basically centered in the use of farnesylated proteins to induce surface photopatterning. Recently, we reported the use of thiol-ene $\mathrm{e}^{31,32}$ and thiol-yne ${ }^{33}$ click reactions to couple monothiolated oligonucleotides onto alkenylated or alkynylated silicon-based surfaces in a direct, clean and quick way. 
1 The obtained DNA microarrays detected bacterial PCR products with high sensitivity and 2 selectivity.

3 Aiming to improve the performance of the fabricated microarrays even more, several 4 important technical issues still remain challenging. These include reducing surface effects

5 such as steric hindrance and electrostatic interactions and controlled arranging of the

6 capture biomolecules in an oriented manner, providing a solution-phase-like environment

7 for biorecognition.

8 Recently, Morvan and colleagues ${ }^{34}$ reported rapid genotyping of hepatitis $\mathrm{C}$ virus using 9 polythiolated probes. These probes developed in this study displayed an increased sensitivity in both in vitro ELOSA on maleimide activated plates and electrochemical assays on gold electrodes.

Here, analogous polythiolated probes are used for the first time on silicon-based materials by thiol-ene and thiol-yne click chemistries to tether the nucleic acids in an optimal

\section{RESULTS AND DISCUSSION}

The process of DNA hybridization at surfaces is a critical part of nucleic acid-based array technology and fundamental understanding of this process under relevant conditions for actual assays is currently very challenging. Thus, controlling probe density on substrates to further optimize probe-target binding kinetics is important.

This will allow to develop new microarray surfaces with better performance within complex media. For the first time, a double control on the microarray performance is exerted by combining surface hydrophobicity tuning and multi-point probe attachment. The modulation of the hybridization capability allows detecting the presence of bacterial DNA and, at the same time, in the same chip, quantifying the microorganism level. Polythiolated oligonucleotides with and without Cy5 dye were obtained on a DNA synthesizer according to standard phosphoramidite chemistry, starting from nucleoside 
or Cy5 solid supports. After elongation of the sequence, the thiol functions were

2 introduced with the same chemistry allowing a straightforward obtaining of mono and polythiolated oligonucleotides. ${ }^{34}$ The crucial point was to remove the cyanoethyl protecting group of the phosphate before deprotection of the thiol functions. Indeed the acrylonitrile formed during classic ammonia treatment strongly reacts with a thiol leading to further unreactive thiol-cyanoethyl. For that purpose, the solid-supported thiolated oligonucleotides were firstly treated with piperidine allowing the selective removal of the cyanoethyl groups. Secondly, after washes, the ammonia treatment was applied for the release from the solid support and the deprotection of the oligonucleotide. Note that the thiol function rapidly oxidized due to oxygen dissolved in solvent leading to a disulfide bridge that should be reduced before immobilization of the mono and polythiolated oligonucleotides on a surface.

Studies in microarray format. Before organosilanization, the silicon oxide chips were tetrathiolated oligonucleotide probes using thiol-ene and thiol-yne coupling chemistries. activated employing a UV-ozone cleaning system. Different exposition times were tried, and water contact angles measured. Finally, an activation time of 7 min was set (Figure S2, Supporting Information.). Immediately after activation, the chips were immersed into a solution of $2 \%$ organosilane in toluene for $2 \mathrm{~h}$, under mild stirring. In the case of alkenyl surfaces, two organosilanes were tried showing similar results, allyltrimethoxysilane and vinyltrimethoxysilane, we decided to use vinyltrimethoxysilane for further studies. In the case of alkynyl derivatization, after silanization, the chips were treated with $2 \%$ propargylamine in toluene for $4 \mathrm{~h}$. The success in the surface functionalization was evaluated by measuring the water contact angle (Figure 1 and Table S2, Supporting Information).

In this way, alkenylated and alkynylated surfaces were ready to immobilize mono, di and 


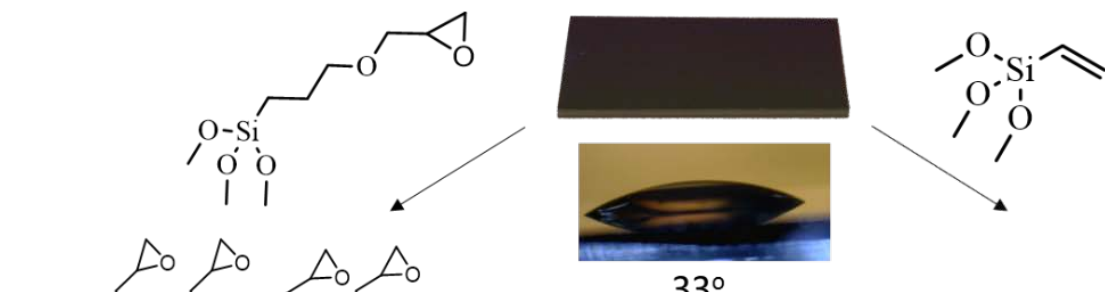

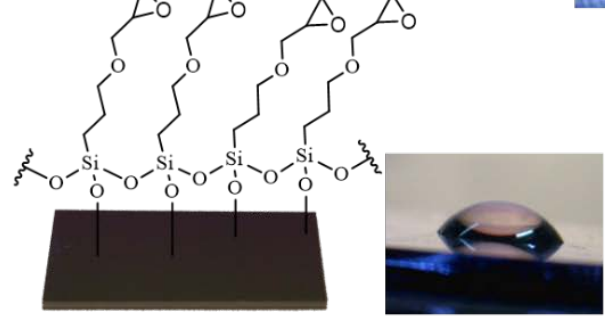

$51^{\circ}$
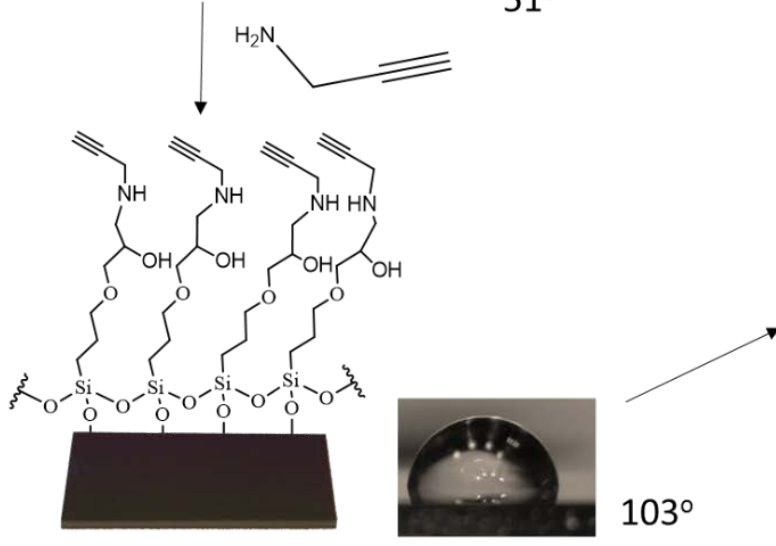
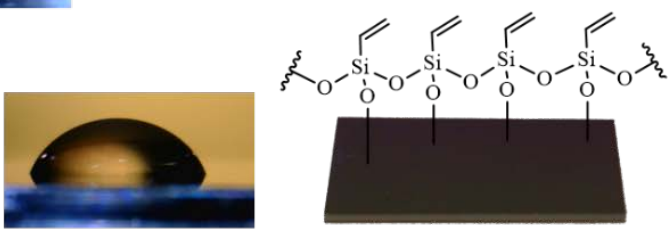

$76^{\circ}$

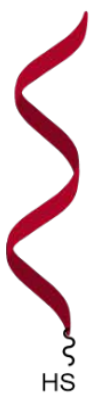

T1.H

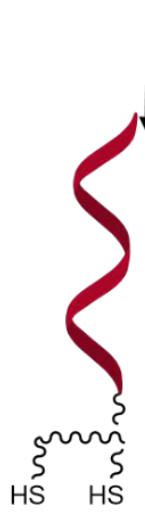

$\mathrm{T} 2 . \mathrm{H}$

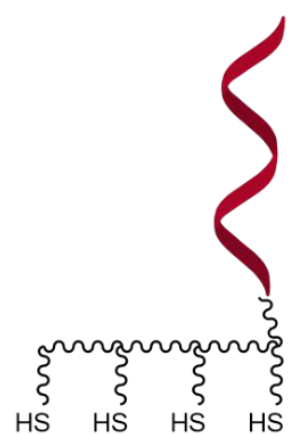

T4.H

Figure 1. Scheme showing the different functionalization approaches providing alkyne and alkene ended surfaces, to attach mono-, di- and tetra-thiolated oligonucleotides. Water contact angles were measured for each surface to assess the progress in the derivatization

Firstly, the mono and polythiolated probes were compared regarding their immobilization capabilities. For this, an array was created onto the functionalized surfaces containing T1.I, T2.I and T4.I at three different concentrations $(0.5,1$ and $2 \mu \mathrm{M})$. T1.I stands for the monothiolated probe, while T2.I and T4.I correspond to the di- and tetra-thiolated probes, respectively. All of them bore a fluorescence tag. Three replicas of each microarray were done and, after irradiating at $365 \mathrm{~nm}$ and washing, the fluorescence was registered and compared. The results are summarized in Figure 2, where immobilized probe density is plotted against the spotted probe concentration for each attachment approach. The amount of immobilized probe was calculated from the decrease in the fluorescence signal after washings, and considering the printed volume $(40 \mathrm{nl})$ and the area of the spots.

The conclusion extracted for alkenyl-terminated surfaces was that polythiolated probes immobilized more effectively on the surface than the monothiolated ones when the probes were spotted at low concentration $(0.5$ and $1 \mu \mathrm{M})$. For alkynyl-ended surfaces, the three probes showed similar immobilization behavior, with no significant differences between 
1 them for concentration of 1 and $2 \mu \mathrm{M}$, while at $0.5 \mu \mathrm{M}$ concentration, the monothiolated 2 probe exhibited a much lower density of immobilization. In all cases, the amount of 3 immobilized probes was higher for thiol-yne coupling chemistry $\left(30.52 \mathrm{pmol} / \mathrm{cm}^{2}\right)$ than 4 for thiol-ene one (13.27 pmol/ $\left.\mathrm{cm}^{2}\right)$ (Table S3, Supporting Information).

Thiol-ene approach

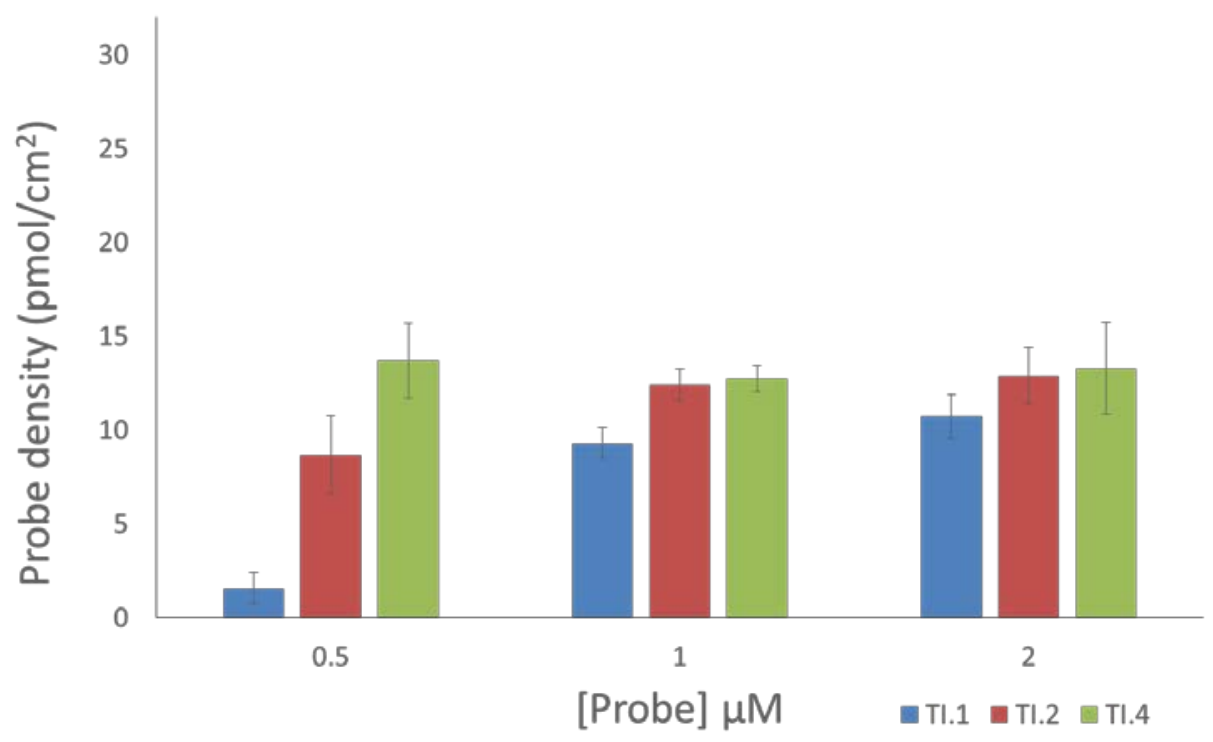

5

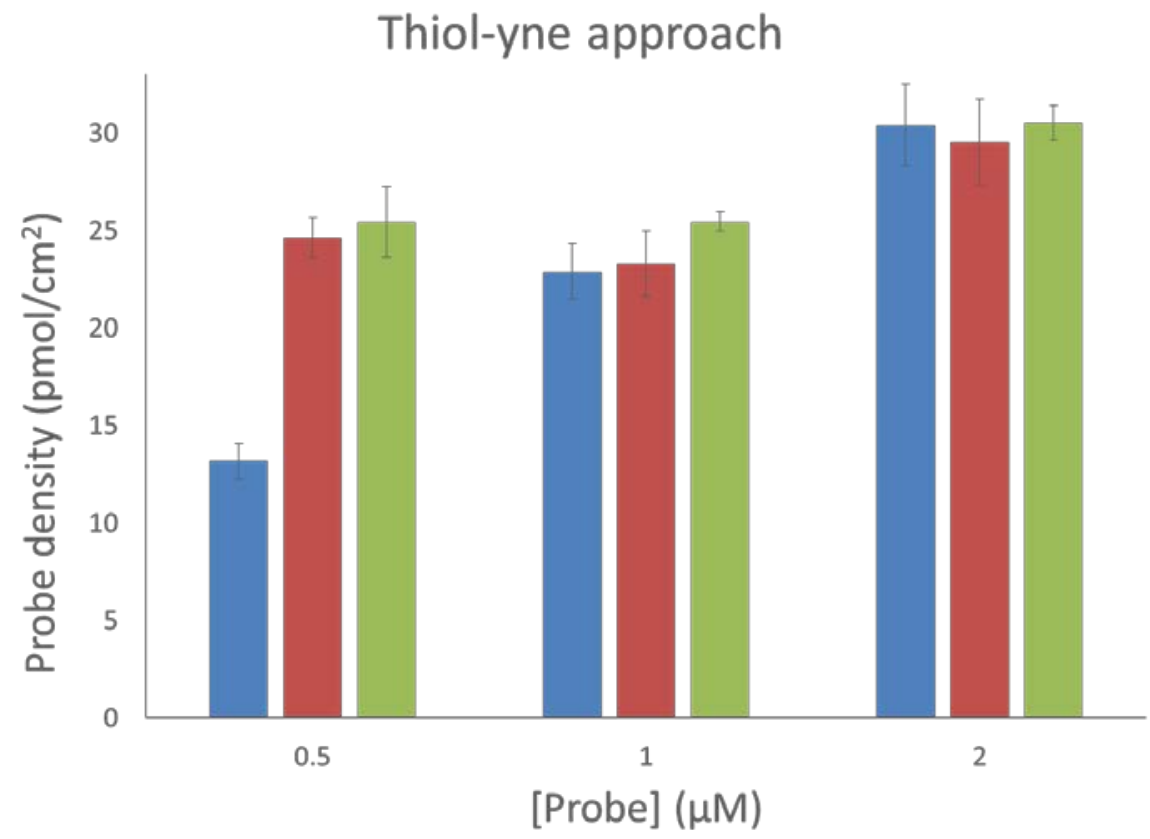

7

8 Figure 2. Immobilized probe density ( $\mathrm{pmol} / \mathrm{cm}^{2}$ ) for mono (blue), di (red) and tetrathiolated (green) 9 oligonucleotides onto alkenylated (thiol-ene coupling: TEC) and alkynylated (thiol-yne coupling: 10 TYC) surfaces after irradiation at $365 \mathrm{~nm}$ for $1 \mathrm{~h}$. 
1 Besides, the use of tetrathiolated probes reached the maximal immobilization density regardless the probe concentration used, whereas for the mono- and di-thiolated probes, higher probe concentrations were needed to achieve maximum immobilization densities. Experiments carried out by Raman spectroscopy and using the Ellman's test did not show evidence of free thiol on the surface after the attachment. However, no conclusive result were obtained. The Ellman's test was not sensitive enough to detect amounts of thiols in the order of our amounts. In the Raman spectra, the presence of other bands from the oligonucleotide structure overlapped the band at $2546 \mathrm{~cm}^{-1}$ specific for free thiols.

As it is known, a higher immobilization density can render less effective hybridization yield. ${ }^{[7]}$ Thus, a new set of chips were functionalized and arrays of probes printed as before, but now using T1.H, T2.H and T4.H. These probes were similar to T1.I, T2.I and T4.I but without the fluorescent tag. Microarrays with growing concentrations of probe (from 0.5 to $5 \mu \mathrm{M}$ ) were printed. After irradiation at $365 \mathrm{~nm}$ for $60 \mathrm{~min}$, and washing, the chips were hybridized with Target A $0.5 \mu \mathrm{M}$ in SSC $1 \times$ for $60 \mathrm{~min}$ at $37^{\circ} \mathrm{C}$. The amount of hybridized oligonucleotide was determined interpolating the fluorescence intensity in the corresponding calibration curve (Figure S3, Supporting Information). What is explained on the basis of the higher surface hydrophobicity, which reduces the contact area and forces the probes to anchor the surface in a denser way.

The hybridization densities were higher for thiol-yne coupling chemistry than for thiolene coupling, indicating that the highest immobilization density still allows for the complementary strand to reach most of the probes, and there is not crowding effects.

Thus, the immobilized probe density was double in thiol-yne than in thiol-ene coupling, and also the hybridization densities. However, the most important feature for our study was that in thiol-ene approach, the multipoint attachment of probes improved significantly the immobilization density and thus the hybridization with the complementary strand (Figure 3a).

From the obtained data of immobilization densities for probes T1.I, T2.I and T4.I at 1 and $2 \mu \mathrm{M}$; and referring them to the values of hybridization density, it was possible to calculate the hybridization efficiency in each case. In Table 1, the estimated hybridization yields are shown. 
a)

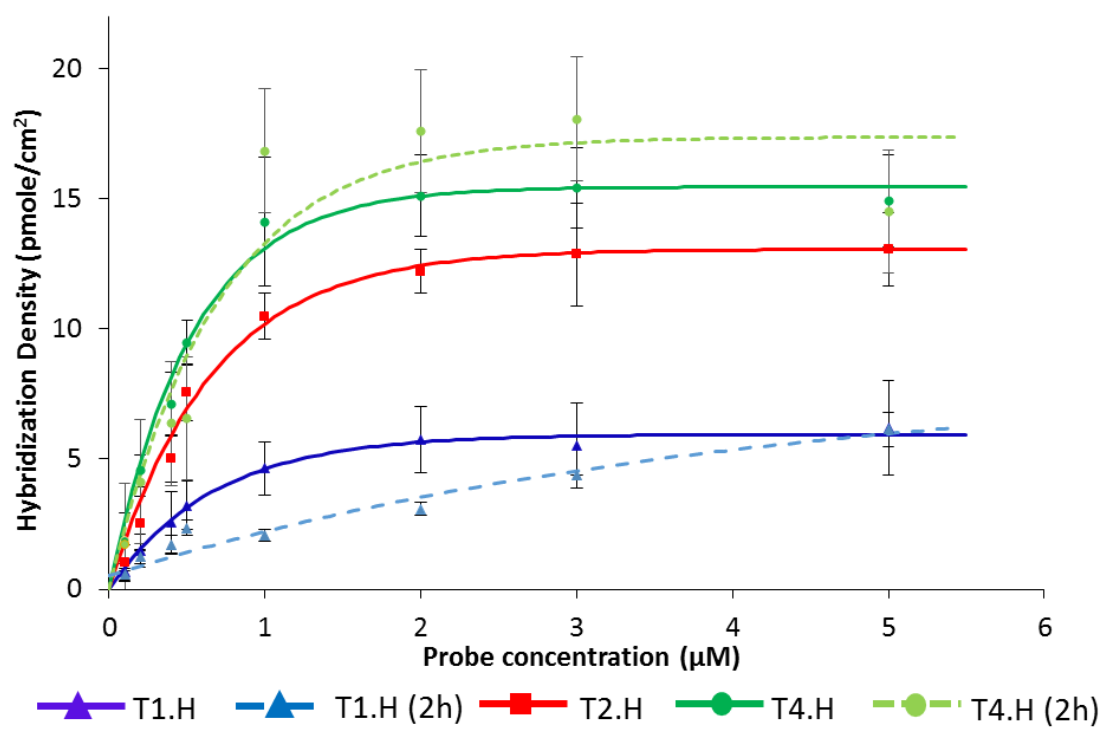

2

3 b)

4

5

6

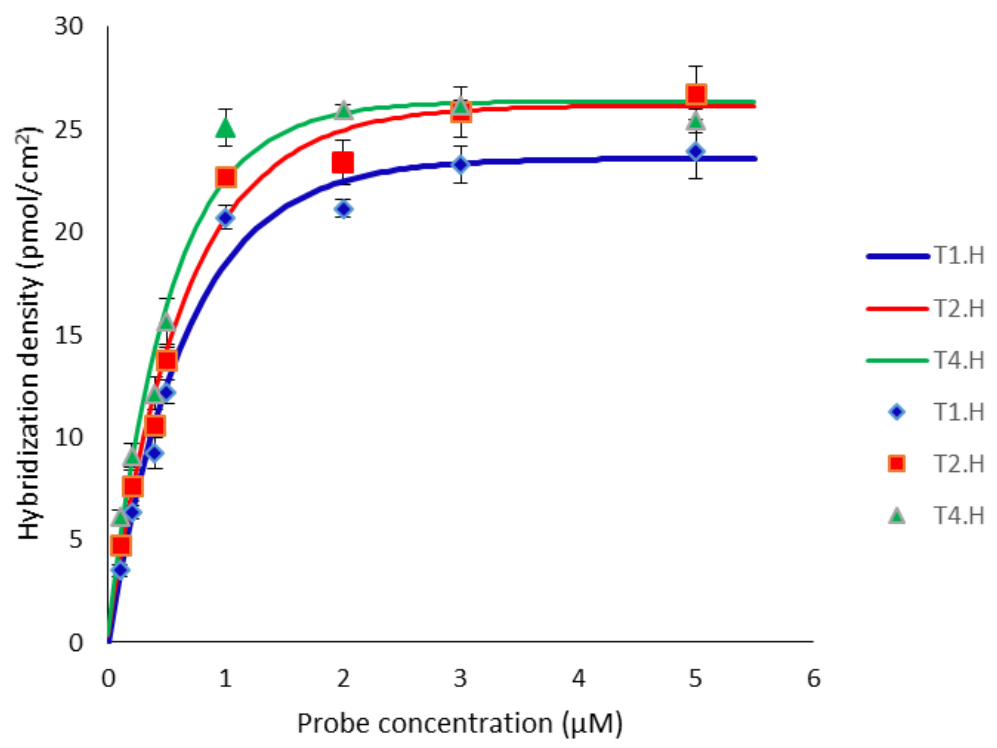

Figure 3. Hybridization densities obtained for Target A $0.5 \mu \mathrm{M}$ in microarrays with growing concentrations of mono- (T1.H), di- (T2.H) and tetra-thiolated (T4.H) probes attached to the surface by means of a) thiol-ene coupling and b) thiol-yne coupling. In a) the dashed lines are for hybridization curves obtained under similar hybridization conditions, but for TH.1 and TH.4 irradiated for $2 \mathrm{~h}$ instead of $1 \mathrm{~h}$.

Regardless of the concentration of spotted probe ( 1 or $2 \mu \mathrm{M})$, the hybridization efficiency increased when the number of thiols contained in the probe grew. This feature was 3 observed for both thiol-ene and thiol-yne approaches. However, it was enhanced in the 14 case of thiol-ene coupling, where the hybridization yield increased from $54 \%$ for T1.H at 
$11 \mu \mathrm{M}$ to $85 \%$ and $100 \%$ for $\mathrm{T} 2 . \mathrm{H}$ and $\mathrm{T} 4 . \mathrm{H}$, respectively. In the case of thiol-yne 2 approach, the effect was less pronounced due to the high yields obtained in all the cases.

3 Thus, yields changed from 90\% for T1.H (1 $\mu \mathrm{M})$ to 98 and 99\% for T2.H and T4.H,

4 respectively. Same pattern was observed at the $2 \mu \mathrm{M}$ concentration.

5 In the case of thiol-ene coupling, longer reaction times did not lead to higher probe 6 immobilization or better hybridization densities. Thus, irradiation times of $2 \mathrm{~h}$ instead of

$7 \quad 1 \mathrm{~h}$ provided hybridization yields very similar to that obtained for $1 \mathrm{~h}$ irradiation, for both

$8 \quad$ TH.1 and TH.4 (Figure 3a, dashed lines)

9 When analyzing the influence of the multipoint attachment in the molecular crowding 10 effect, higher differences between thiol-ene and thiol-yne coupling were appraised. Thus, when comparing the hybridization percentages under saturation of probe, changing from 1 to $2 \mu \mathrm{M}$, for T1.H, meant a decrease in hybridization efficiency, which lowered from $53 \%$ to $42 \%$. This fact, although, was not noticed for T2.H and T4.H, which even increased the hybridization yields (from 85 to $96 \%$ in T2.H). T4.H kept the maximal hybridization efficiency for both probe concentrations (Table 1).

Table 1: Hybridization percentage referred to the immobilized density for a probe concentration of 1 and $2 \mu \mathrm{M}$, for thiol-ene and thiol-yne approaches and using mono-, di- and tetra-thiolated probes.

\begin{tabular}{|c|c|c|c|c|c|c|}
\hline \multirow[t]{2}{*}{ Probe conc. $(\mu \mathrm{M})$} & \multicolumn{3}{|c|}{ Thiol-ene approach } & \multicolumn{3}{|c|}{ Thiol-yne approach } \\
\hline & T1.I & T2.I & T4.I & T1.I & T2.I & T4.I \\
\hline & \multicolumn{6}{|c|}{ Immobilized density $\left(\mathrm{pmol} / \mathrm{cm}^{2}\right)$} \\
\hline 1 & 8.7 & 12.4 & 12.9 & 22.9 & 23.3 & 25.5 \\
\hline \multirow[t]{2}{*}{2} & 13.7 & 12.7 & 13.3 & 30.4 & 29.5 & 30.5 \\
\hline & \multicolumn{6}{|c|}{ Hybridization density $\left(\mathrm{pmol} / \mathrm{cm}^{2}\right)$} \\
\hline 1 & 4.6 & 10.5 & 14.1 & 20.7 & 22.7 & 25.1 \\
\hline \multirow[t]{2}{*}{2} & 5.7 & 12.2 & 15.1 & 21.2 & 23.4 & 26.0 \\
\hline & \multicolumn{6}{|c|}{ Hybridization yield (\%) } \\
\hline 1 & 54 & 85 & 100 & 90 & 98 & 99 \\
\hline 2 & 42 & 96 & 100 & 70 & 79 & 85 \\
\hline
\end{tabular}

The molecular crowding effect was also noticed using the monothiolated probe in thiolyne coupling surfaces, lowering the yield from 90 to 70\%, when spotted concentrations of T1.H moved from 1 to $2 \mu \mathrm{M}$. Di- and tetra-thiolated probes showed also a slight crowding effect. However, it was much lower than in the case of the monothiolated probe. 
1 Thus, the hybridization yield decreased from 98 to $79 \%$ in T2.H, and from 99 to $85 \%$ in

2 T4.H. To assess reproducibility, the assays were done in triplicate, and repeated on

3 different days. The intrachip RSD oscillated between 5\% and 12\%, meanwhile interchip

$4 \quad$ RSD was in the range from $12 \%$ to $15 \%$.

$5 \quad$ AFM and XPS studies on alkene biofunctionalized chips were also performed before and

6 after hybridization (Figures S4 and S5, Supporting Information). The results agreed with

7 that observed in the microarrays, the amount of immobilized probe was higher for di and

8 tetrathiolated probes than for monothiolated one.

9 As conclusion, the use of di- and tetra-thiolated improved the performance of the hybridization, especially in the case of thiol-ene coupling surfaces or when the crowding effect acted. Briefly, there are two ways to improve the performance of a microarray: to focus on the surface functionalization and tune its features, or to link the probe using a multipoint attachment. Both options seem to be closely related to the configuration adopted by the probe once attached, which determines its bioavailability, and which is influenced by the properties of the surface itself (hydrophobicity, etc) and the anchoring way.

In order to look more deeply in the hybridization process for the different situations, a complete study was done using dual polarization interferometry (DPI). In this technique, the hybridization is monitored label-free in real time and thus, data about the mass surface density, the change in thickness and density are obtained. This can give some light on how the immobilized probes are set in each case, and the changes that they experience after hybridization.

For that purpose, unmodified Anachips (containing two channels available for measurements) were derivatized with alkenyl or alkynyl groups. Taking advantage of our immobilization chemistry, the chips were functionalized with a different thiolated probe on each channel, using selective irradiation through a homemade photomask. Thus, a set of four chips were ready for DPI studies containing the following pairs of probes immobilized in the channels: Probes T1.H vs T2.H as well as T1.H vs T4.H by thiol-ene coupling chemistry and the pairs T1.H vs T2.H and T1.H vs T4.H anchored by thiol-yne coupling chemistry. In all the cases, the concentration of probe was $1 \mu \mathrm{M}$. For each chip the experiment was the same, after flowing hybridization buffer (SSC $1 \times$ ), Target B at 5 $\mu \mathrm{M}$ was injected in both chips and flowed over for $25 \mathrm{~min}$ (Figure S6, Supporting Information). After flowing buffer for several min, water was injected to dehybridize and a non-complementary strand was later flowed in order to assess the specificity in the 
1 hybridization. From the transverse electric (TE) and transverse magnetic (TM) plots,

2 quantitative data were extracted, as mass density, volume density, refractive index 3 variations, and layer thickness.

4 Considering the immobilization density obtained from the microarray assays for $1 \mu \mathrm{M}$

5 probe concentration, hybridization efficiencies were calculated in each case and

6 compared with those obtained in microarray format. Trends observed in these DPI

7 experiments were in agreement with the observed in microarrays. For thiol-ene coupling

8 approach, the density of hybridized oligonucleotide rose as the number of thiol moieties

9 in the probe increased. However, for thiol-yne immobilization, the hybridization

10 efficiency remained constant regardless the number of thiols present in the probe. Interestingly, the same ratio of improvement in the hybridization efficiency was observed for both microarray assays and DPI experiments in thiol-ene coupling and thiol-yne coupling plots, when the number of thiols in the probes grew.

Table 2: DPI figures obtained for thiol-ene and thiol-yne coupling for T1.H, T2.H and T4.H after hybridization with Target B $0.5 \mu \mathrm{M}$.

\section{Thiol-ene}

\section{Thiol-yne}

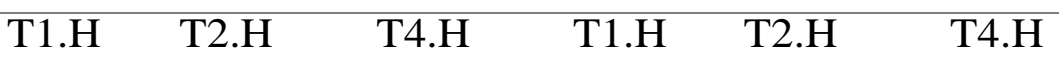




\begin{tabular}{lllllll}
\hline Refractive Index & 1.36 & 1.41 & 1.43 & 1.49 & 1.50 & 1.53 \\
\hline Thickness (nm) & 0.33 & 0.34 & 0.29 & 0.28 & 0.32 & 0.39 \\
\hline Mass (ng/mm²) & 0.04 & 0.14 & 0.15 & 0.24 & 0.30 & 0.42 \\
\hline Density (g/cm $\left.{ }^{3}\right)$ & 0.11 & 0.42 & 0.53 & 0.87 & 0.94 & 1.06 \\
\hline Mass* $\left(\mathbf{p m o l} / \mathbf{c m}^{2}\right)$ & 0.50 & 1.89 & 2.16 & 3.37 & 4.18 & 5.87
\end{tabular}

*Calculated from the mass surface and considering a molecular weight for Target B of 7,127 g/mol

Considering the theoretical density of a double stranded DNA, $1.7 \mathrm{~g} / \mathrm{cm}^{3}$, the obtained densities would correlate with the following percentages of dsDNA after hybridization having one, two and four thiol groups in the probe, respectively: 6\%, 25\% and 31\% for thiol-ene coupling approach, and 51\%, 55\%, and 62\% for thiol-yne strategy (Table 3).

Table 3: Probe immobilized density obtained from microarray assays, and hybridization efficiencies obtained in DPI experiments calculated considering the immobilized probe and the theoretical density of a double stranded DNA.

\begin{tabular}{|c|c|c|c|c|c|c|}
\hline & \multicolumn{3}{|c|}{ Thiol-ene } & \multicolumn{3}{|c|}{ Thiol-yne } \\
\hline & T1.I & T2.I & T4.I & T1.I & T2.I & T4.I \\
\hline Immobilized density $\left(\mathrm{pmol} / \mathrm{cm}^{2}\right)^{\mathrm{a}}$ & 8.08 & 12.6 & 13.11 & 22.9 & 23.3 & 25.5 \\
\hline Hybridization yield ${ }^{\mathrm{b}}$ (\%) & 6 & 15 & 17 & 12 & 15 & 20 \\
\hline Hybridization yield ${ }^{c}(\%)$ & 6 & 25 & 31 & 51 & 55 & 62 \\
\hline
\end{tabular}

${ }^{\mathrm{a}}$ Microarray data for $1 \mu \mathrm{M}$ of probe, ${ }^{\mathrm{b}}$ calculated using the mass obtained in DPI, and the immobilized density determined by microarray ${ }^{\mathrm{c}}$ calculated using the density obtained in DPI and the theoretical density of a double stranded DNA.

Nevertheless, taking into account that the amount of immobilized probe by thiol-ene coupling was half the immobilized probe reached by thiol-yne approach, we concluded that the four-thiol attachment enhances the performance in hybridization of thiol-ene coupling strategy, reaching the level of efficiency of thiol-yne coupling. This indicates that the control in the solid-probe-fluid interface can be done by using different surface chemistries, or by using probe multi-point attachment as well.

DPI experimental data suggest that the probes stand up in all cases for thiol-yne coupling attachment, while in the case of thiol-ene coupling attachment, the monothiolated probe lays down on the surface, and polythiolated probes stand up on the surface. This is determined by the theoretical thickness increase considering a perfect close packed dsDNA layer on the surface (when the surface coverage is less than $20 \%$, the provided thickness is the averaged thickness, that is $0.20 \times$ Thickness dsDNA). Thus, when dividing the obtained experimental thickness by the hybridization percentage, the theoretical 
1 thickness obtained resulted $2 \mathrm{~nm}$ for all cases, except for the case of thiol-ene coupled

2 monothiolated probe, whose thickness resulted $5.5 \mathrm{~nm}$. This indicates that the probe, in

3 the last case has been straightened much more that in the other cases, which means that it

4 was much more tilted, laying down on the surface. This would difficult target

5 accessibility, diminishing then the hybridization capability.

6 It is worth noticing that DPI usually yields worse hybridization than microarray because

7 the incubation time is shorter, 25 min instead of $1 \mathrm{~h}$, and the flow can negatively affect

8 the hybridization process.

9 In Figure 4, the Transverse Electric (TE) variation is plotted is shown for hybridization of T1.H, T2.H and T4.H attached by thiol-ene coupling. The evolution of TE follows the same trend in the three cases but the change in TE is bigger as the number of thiols in the probe increases.

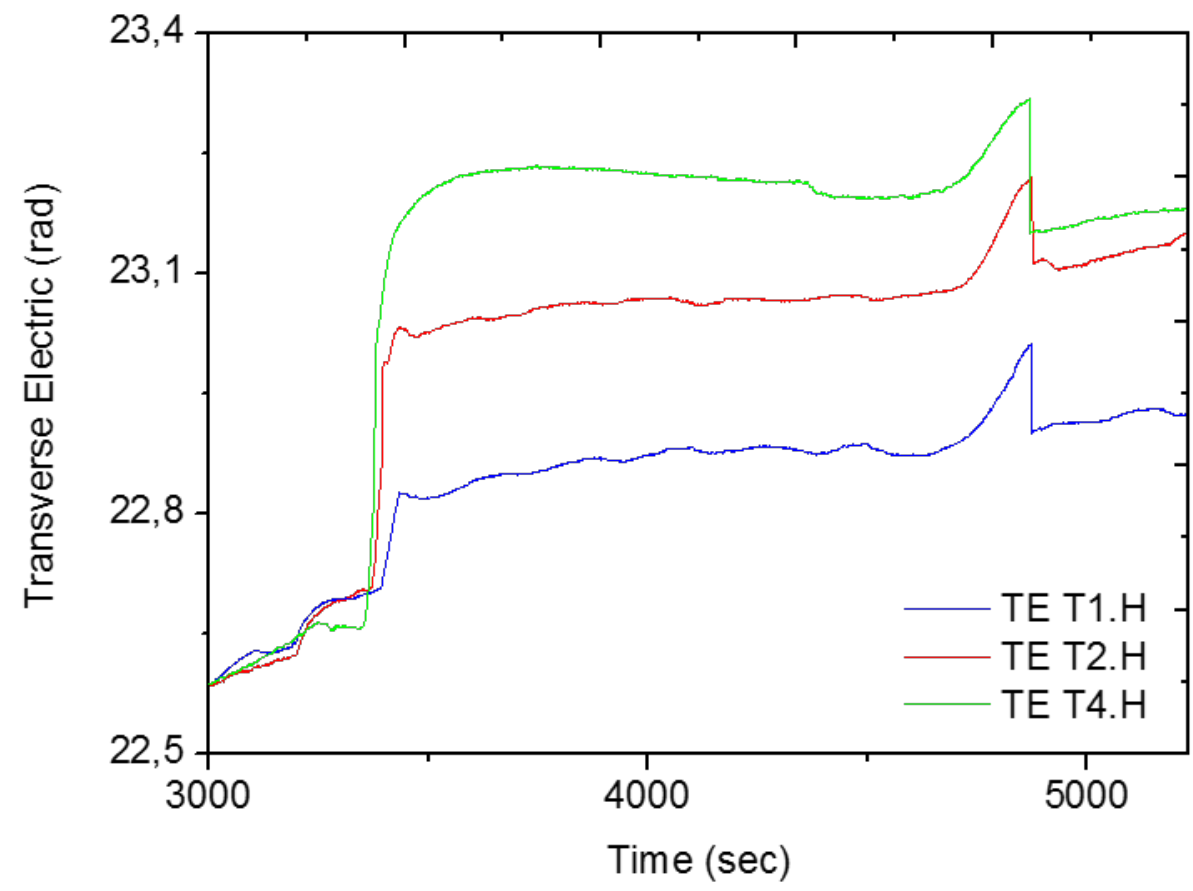

13

Figure 4: Transverse Electric evolution during hybridization of $0.5 \mu \mathrm{M}$ of Target B in DPI for immobilized probes T1.H, T2.H and T4.H $(1 \mu \mathrm{M})$. Black arrows indicate the start and the end of the Target B injection in the channels.

\section{PCR products detection}

Finally, in order to demonstrate the applicability of the developments for real samples, further experiments were done to detect PCR products of an innocuous specie of Salmonella. In this case, glass was used as solid support instead of silica. The reason was 
to assay colorimetric detection, which would allow naked eye identification without any instrumental detection.

The functionalization proceeded in the same way as silica, as glass surfaces respond also very well to organosilane functionalization. Three glass chips were functionalized with vinyl triethoxysilane as before. Then each array was printed with the probes specific for Salmonella T1.Sal, T2.Sal, and T4.Sal, containing one, two and four thiol groups, respectively. Two sequences were also printed: the T1.I as immobilization control and the T4.Cam targeting Campylobacter as a probe specificity control. Hybridization was carried out for $1 \mathrm{~h}$ at $37^{\circ} \mathrm{C}$ with a 1/10 dilution of the PCR products corresponding to a 500 pM concentration.

After hybridization with Salmonella digoxigenin-labeled PCR products, two chips were incubated with a mixture containing anti-digoxigenin rabbit antibody (1/10000) and goldlabeled goat anti-rabbit antibody (1/100). The microarrays were then developed with silver enhancer solution, showing a black precipitate only in the rows corresponding to T1.Sal, T2.Sal and T4.Sal (Figure 5a).
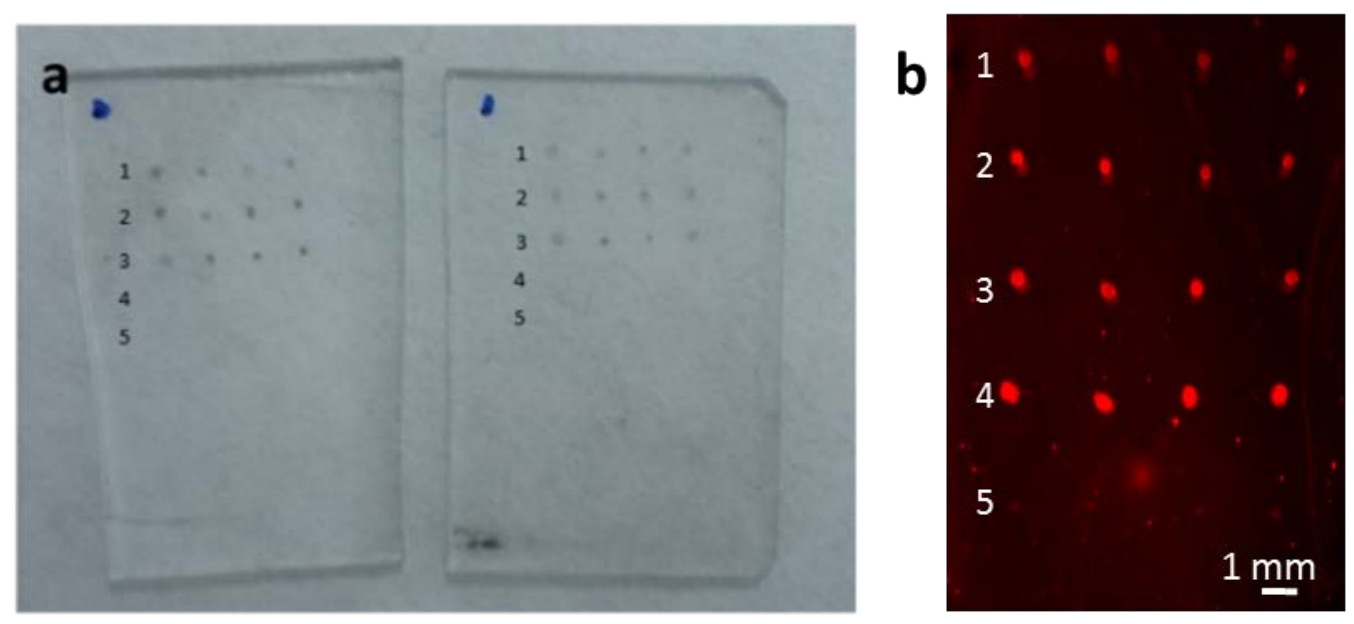

Figure 5. Microarrays on glass after hybridization with Salmonella PCR products. a) Colorimetric detection using silver development format and b) Fluorescence detection. First row corresponds to T1.Sal, row 2 corresponds to T2.Sal, row 3 corresponds to T4.Sal, row 4 is T1.I, and row 5 corresponds to T4.Cam, both controls.

The third chip was treated, after PCR products hybridization, with anti-digoxigenin rabbit antibody (1/100) in PBS-T for $30 \mathrm{~min}$, washed with water and incubated again with Alexa647-labeled goat anti-rabbit antibody 1/50 in PBS-T for another 30 min. After washing, the fluorescence was measured (Figure 5b). Fluorescence signal could be 
1 observed for the rows T1.Sal, T2.Sal and T4.Sal, and for control T1.I as well.

2 Fluorescence labelling allowed detection and quantification of the signal reached for each

3 probe. We observed that the dithiolated probe enhanced the signal $10 \%$ related to the

4 monothiolated T1.Sal, while tetrathiolated probe raised the signal up to $26 \%$ (Table S4,

5 Supporting Information). Although good sensitivity was obtained for all probes, it was

6 demonstrated again that multipoint probe attachment improved the hybridization

7 efficiency, even for large DNA fragments such the current PCR products (150 bp). In

8 addition, no hybridization was observed with the T4.Cam probe, demonstrating the

9 selectivity of the hybridization and the absence of non-specific immobilization on the 10 chip.

11 Using colorimetric detection, further experiments with more diluted PCR products (from

$121 / 10$ to $1 / 100$ ) were done. Hybridization was detected up to dilution $1 / 40$, which 13 corresponds to a concentration of $125 \mathrm{pM}$.

14 For dilutions below 1/40, only T4.Sal showed positive results. Thus, serial dilutions were 15 done and assayed for the tetrathiolated probe, in order to determine the lowest concentration to be detected using the most sensitive probe (Figure S7, Supporting Information). Under these conditions, the probe hybridized with dilutions up to $1 / 240$, which means a concentration of $20 \mathrm{pM}$. The selectivity of the probe of this concentration level was assessed including a control row with a tetrathiolated probe specific for Campylobacter bacteria. This probe didn't develop positive assay for Salmonella PCR products but did for Campylobacter PCR products at 1/100.

22 As final demonstration of the applicability of the method proposed herein, a fluorescence23 based microarray assay was designed. In it T1.Sal, T4.Sal, T1.Cam, T4.Cam and T1.I were immobilized as depicted in Figure 6a). 


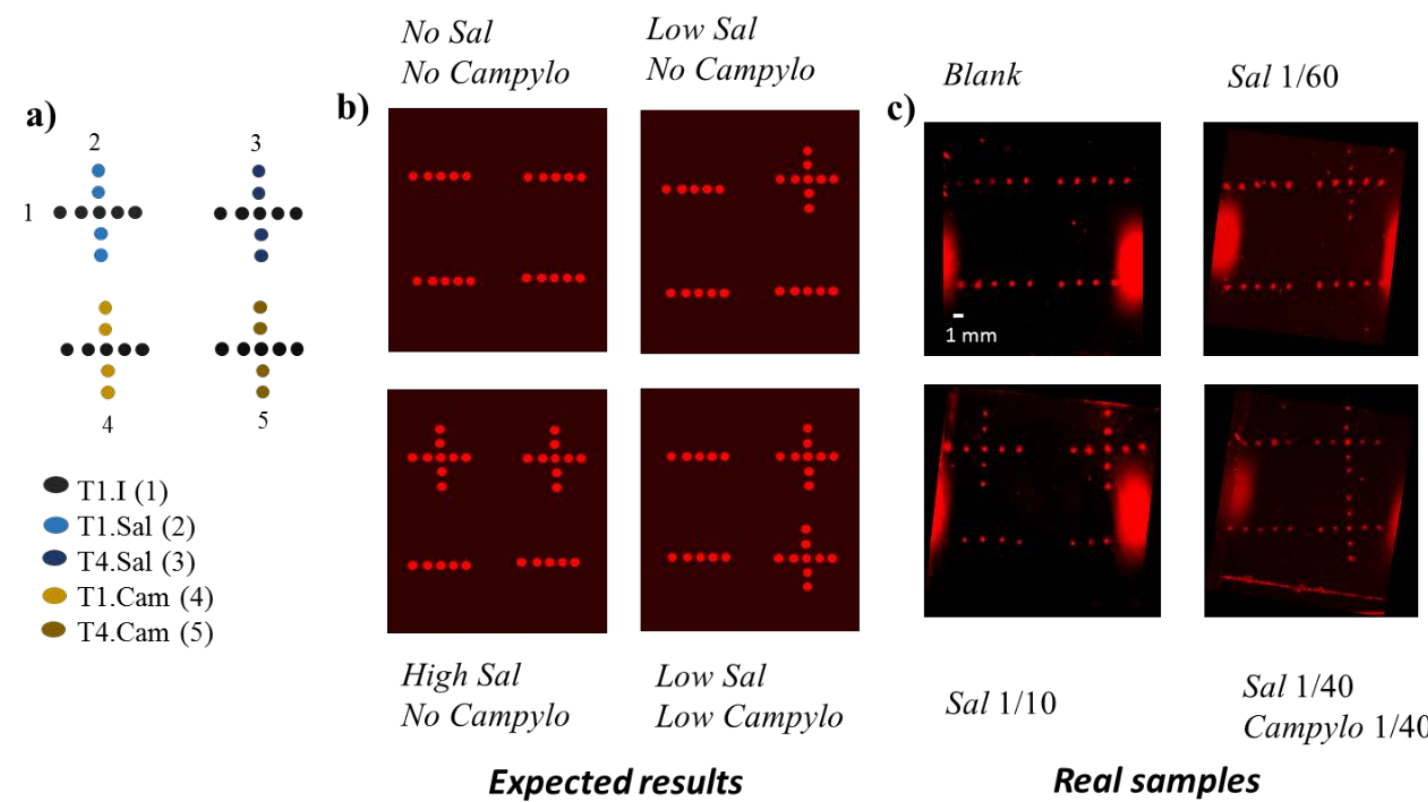

Figure 6. a) Scheme of the designed microarray where rows are printed with immobilization control probe T1.I, whereas columns are printed with monothiolated (2) and tetrathiolated (3) probes for Salmonella and monothiolated (4) and tetrathiolated (5) probes for Campylobacter (1 $\mu \mathrm{M})$ b) expected results for different situations with low or high concentrations of bacterial DNA in samples c) obtained results for samples without bacterial DNA (top-left), with low (top-right) and high (bottom-left) concentration of Salmonella's DNA and with a mixture of Salmonella and Campylobacter PCR products (bottom-right).

This design would allow easy differentiation of samples containing higher and lower concentrations of Salmonella, and the same for Campylobacter (Figure 6b). These arrays were prepared and assayed (by duplicate) with samples containing PCR products of Salmonella and/or Campylobacter at different concentrations. The results obtained are shown in Figure 6c, where two plus marks were obtained for Salmonella 1/10, while only one plus mark was observed for dilution 1/60. The number and location of plus marks indicated the bacteria specie present in the sample (Salmonella, Campylobacter or both) and the concentration level (two plus marks for dilution up to 1/40, and only one mark for higher dilutions).

\section{CONCLUSION}

In this work, thiol-ene and thiol-yne coupling chemistries have been evaluated to attach mono and polythiolated probes onto alkenylated and alkynylated surfaces, respectively. Studies tackled by dual polarization interferometry and on chip microarray fluorescence format indicated that alkynyl terminated surfaces rendered higher immobilization yields than thiol-ene linking. Polythiolated probes were more effectively immobilized on the 
1 surface than the monothiolated ones. Closely related to the immobilized probe, the

hybridization density was also double in thiol-yne approach. However, it was observed with the thiol-ene coupling chemistry that multipoint probe attachment improved significantly the immobilization density and thus, the hybridization yield with the complementary strand. This trend was also observed for thiol-yne coupling although less pronounced. Also, for hybridization of large DNA strands, such as real bacterial PCR products, the same behavior was noticed and detection was improved using multi-point attachment in thiol-ene approach.

Consequently, there are two ways to improve the performance of a microarray; the first one is to focus on the surface functionalization, tuning surface properties such as hydrophobicity, and the second one is to control the surface-probe-fluid interface by multi-point probe attachment. Both approaches seem to be closely related to the configuration adopted by the attached probe, leading to its good availability for hybridization with

PCR product. Considering these issues together when designing new microarrays could help to reach advanced performance in the hybridization assays.

As demonstrated in the experiments, the created microarrays can be used with both colorimetric and fluorescence detection techniques. The first provides higher sensitivity; however, the second presents the advantages of lower number of steps and rapid readout. The flexibility in the detection approach would allow the development of an assay where the presence and concentration of bacterial DNA would be read by the naked eye.

\section{EXPERIMENTAL SECTION (Experimental Section has been moved behind}

\section{Conclusion section)}

Chemicals, Reagents, and Buffers. Silicon-based wafers were provided by Valencia Nanophotonics Technology Center (NTC) at Universitat Politècnica de València (Spain) from SIEGERT WAFER GmbH (Aachen, Germany) as a $2 \mathrm{~mm}$ thick silicon oxide layer grown on a (1 00 ) silicon wafer. Glass microscope slides were obtained from Labbox (Barcelona, Spain).

Allyltrimethoxysilane, vinyl trimethoxysilane, (3-glycidyloxypropyl)trimethoxysilane (GOPTS), propargylamine, tris(2-carboxyethyl)phosphine hydrochloride (TCEP), and silver developer solutions A and B were purchased from Sigma-Aldrich Química 
1 (Madrid, Spain). Toluene, 2-propanol, and formamide were purchased from Scharlau

2 (Madrid, Spain).

3 Oligonucleotide sequences Target $\mathrm{A}$ and Target $\mathrm{B}$ were acquired from Eurofins

4 Genomics (Ebersberg, Germany). Monothiolated oligonucleotide sequences T1.I, T1.H and T1.Cam were acquired from Aldrich Quimica (Madrid, Spain).

Polythiolated-modified probes T2.I, T4.I, T2.H, T4.H, T1.Sal, T2.Sal, T4.Sal, T4.Cam (Table 4) were synthesized on a $1 \mu \mathrm{mol}$-scale by standard phosphoramidite chemistry using a 394 ABI DNA synthesizer. Cy5 solid support was purchased from Link Technologies (Lanarkshire, Scotland). For the coupling step, benzylmercaptotetrazole (BMT) was used as the activator $\left(0.3 \mathrm{M}\right.$ in anhydrous $\left.\mathrm{CH}_{3} \mathrm{CN}\right)$ along with commercially available nucleoside phosphoramidites ( $\mathrm{dT}, \mathrm{dABz}, \mathrm{dCBz}$ and $\mathrm{dGtBuPac}$ ) at $0.075 \mathrm{M}$ in anhydrous $\mathrm{CH}_{3} \mathrm{CN}$ introduced with a 20 s coupling time,1-O-(4,4'-dimethoxytrityl)-2-(6S-acetylthio hexyl oxymethyl)-2-methyl-3-(diisopropylamino $\beta$-cyanoethyl phosphoramidite)-propane-1,3-diol ${ }^{[27]}$ now commercially available from Chemgenes Corporation (0.1 $\mathrm{M}$ in anhydrous $\left.\mathrm{CH}_{3} \mathrm{CN}\right)$ with a $60 \mathrm{~s}$ coupling time. The capping step was performed with phenoxyacetic anhydride using commercial solutions (Cap A: $\mathrm{Pac}_{2} \mathrm{O}$, pyridine, THF 10/10/80 and Cap B: 10\% N-methylimidazole in THF) for 60 s. Oxidation was performed with a commercial solution of iodide (0.1 M I2, THF/pyridine/water 90/5/5) for 13 s. Detritylation was performed with 3\% TCA in $\mathrm{CH}_{2} \mathrm{Cl}_{2}$ for $65 \mathrm{~s}$.

Table 4. Oligonucleotide sequences list, including functionalities.

\begin{tabular}{llll}
\hline Name & Sequence (5' to 3') & 5' end & 3' end \\
\hline T1.I & CCCGATTGACCAGCTAGCATT & 1 SH & Cy5 \\
\hline T2.I & CCCGATTGACCAGCTAGCATT & $2 \mathrm{SH}$ & Cy5 \\
\hline T4.I & CCCGATTGACCAGCTAGCATT & 4 SH & Cy5 \\
\hline T1.H & CCCGATTGACCAGCTAGCATT & $1 \mathrm{SH}$ & \\
\hline T2.H & CCCGATTGACCAGCTAGCATT & $2 \mathrm{SH}$ & \\
\hline T4.H & CCCGATTGACCAGCTAGCATT & $4 \mathrm{SH}$ & \\
\hline Target A & AATGCTAGCTGGTCAATCGGG & $\mathrm{Cy5}$ & \\
\hline Target B & AATGCTAGCTGGTCAATCGGG & & \\
\hline T1.Sal & T4GATTACAGCCGGTGTACGACCCT & $1 \mathrm{SH}$ & \\
\hline T2.Sal & T4GATTACAGCCGGTGTACGACCCT & $2 \mathrm{SH}$ & \\
\hline T4.Sal & T4GATTACAGCCGGTGTACGACCCT & 4 SH & \\
\hline T1.Cam & T4AGACGCAATACCGCGAGGTGGAGCA & 1 SH & \\
\hline T4.Cam & T4AGACGCAATACCGCGAGGTGGAGCA & 4 SH & \\
\hline
\end{tabular}


1 Protocol for deprotection. After elongation, the solid-supported S-acetylthiololigonucleotides were treated with a solution of $10 \%$ piperidine in dry $\mathrm{CH}_{3} \mathrm{CN}$ in a continuous flow manner ( $5 \mathrm{~mL}$ over $15 \mathrm{~min}$ ), before being washed with dry $\mathrm{CH}_{3} \mathrm{CN}$ and dried using a flush of nitrogen. Then, solid-supported thiolated oligonucleotides were treated with concentrated ammonia for $2 \mathrm{~h}$ at room temperature. The filtrate was withdrawn and evaporated affording the polythiolated probes. The residue was dissolved in $1 \mathrm{~mL}$ of water and washed three times with ethyl acetate to remove benzamide and tert-butylphenoxyacetamide. After MALDI-TOF characterization (Table S1), the crude modified oligonucleotides were lyophilized and stored at $-20^{\circ} \mathrm{C}$. The structure of the thiolated probes can be seen in Figure S1 (Supporting Information)

Milli-Q water $18 \mathrm{~m} \Omega$ was used to prepare aqueous solutions. The buffers employed, phosphate buffer saline (PBS $1 \times$, 0.008M sodium phosphate dibasic, $0.002 \mathrm{M}$ sodium phosphate monobasic, $0.137 \mathrm{M}$ sodium chloride, $0.003 \mathrm{M}$ potassium chloride, $\mathrm{pH}$ 7.5), PBS-T (PBS 10× containing 0.05\% Tween 20), saline sodium citrate (SSC 10×, $0.9 \mathrm{M}$ sodium chloride, $0.09 \mathrm{M}$ sodium citrate, $\mathrm{pH}$ 7) and washing solutions were filtered through a $0.22 \mu \mathrm{m}$ pore size nitrocellulose membrane from Whatman GmbH (Dassel, Germany) before use.

Digoxigenin-labeled PCR products from Salmonella were obtained in the laboratory, as previously described, ${ }^{[35,36]}$ with a concentration of $546.38 \mathrm{ng} / \mathrm{ml}(5 \mathrm{nM})$ determined by fluorescence.

Anti-digoxigenin recombinant monoclonal antibody from rabbit and goat anti-rabbit Alexa Fluor 647 antibody were purchased from Invitrogen Life Technologies (Carlsbad, CA). Gold labeled goat anti-rabbit was ordered from Sigma-Aldrich (Madrid, Spain).

Instrumental methods. Surface activation was carried out with a UV-Ozone cleaning system UVOH150 LAB (FHR, Ottendorf-Okrilla, Germany).

Microarrays were printed with a low volume noncontact dispensing system from Biodot (Irvine, CA), model AD1500.

Probe photoattachment was done with a mercury capillary lamp Jelight $\left(6 \mathrm{~mW} / \mathrm{cm}^{2}\right.$, Jelight Irvine, CA).

Contact angle measurements were carried out with Dino-Lite Microscope and image treated with Dino Capture software (Torrance, CA). The measurements were done in triplicate at room temperature with a volume drop of $5 \mu 1$ employing $18 \mathrm{~m} \Omega$ water quality. The fluorescence signal of the spots in the microarrays was registered with a homemade surface fluorescence reader (SFR), ${ }^{37}$ having a high sensitivity charge coupled device 
camera Retiga EXi from Qimaging, Inc. (Burnaby, Canada), with light emitting diodes Toshiba TLOH157P as light source. Microarray image treatment and quantification was done using GenePix Pro 4.0 software from Molecular Devices, Inc. (Sunnyvale, CA).

Dual Polarization Interferometry studies were carried out with an Analight2000 device (Biolin Scientific, Stockholm, Sweden). Raw silicon oxynitride Anachips (Biolin Scientific) were employed and biofunctionalized as required in each case.

MALDI-ToF mass spectra were registered on a Voyager mass spectrometer (Perspective Biosystems, Framingham, MA) equipped with a nitrogen laser. MALDI conditions were: accelerating voltage $24000 \mathrm{~V}$; guide wire $0.05 \%$ of the accelerating voltage; grid voltage $94 \%$ of the accelerating voltage; delay extraction time $700 \mathrm{~ns} .1 \mu \mathrm{L}$ of sample was mixed with $5 \mu \mathrm{L}$ of a saturated solution of THAP in acetonitrile/water (1:1, v/v) containing $10 \%$ of ammonium citrate and few beads of DOWEX 50W-X8 ammonium sulfonic acid resin were added. Then, $1 \mu \mathrm{L}$ of the mixture was placed on a plate and dried at room temperature and pressure.

X-ray photoelectron spectra were recorded with a Sage 150 spectrophotometer from SPECS Surface Nano Analysis GmbH (Berlin, Germany). Non-monochromatic Al Ka radiation (1486.6 eV) was used as the X-ray source operating at $30 \mathrm{eV}$ constant pass energy for elemental specific energy binding analysis. Vacuum in the spectrometer chamber was $9 \times 10^{-9} \mathrm{hPa}$ and the sample area analyzed was $1 \mathrm{~mm}^{2}$. Atomic Force Microscopy (AFM) measurements were carried out with a Veeco model Dimension 3100 Nanoman from Veeco Metrology, (Santa Barbara, CA) using tapping mode at $300 \mathrm{kHh}$. Imagining was performed in AC mode in air using OMCL-AC240 silicon cantilevers (Olympus Corporation, Japan). The images were captured using tips from Nano World with a radius of $8 \mathrm{~nm}$. All AFM images were processed with WSxM software. ${ }^{38}$

Surface chemical modification. Silicon wafers were cut into pieces of $2 \times 1 \mathrm{~cm}^{2}$, cleaned with water first, then with 2-propanol and blow dried. Afterwards, they were placed in the UV-ozone cleaner, and irradiated for $7 \mathrm{~min}$. The chips were functionalized immediately after activation.

For alkenylation, activated chips were introduced into a solution of vinyltrimethoxy silane ( $2 \% \mathrm{v} / \mathrm{v}$ in toluene) for $2 \mathrm{~h}$ at room temperature. The chips were cleaned with toluene, then with 2-propanol, and blow dried with compressed air. Then they were baked at $150{ }^{\circ} \mathrm{C}$ in an oven for $30 \mathrm{~min}$. 
1 To introduce the alkynyl groups, the chips were immersed under argon atmosphere into 2 a solution of (3-glycidyloxypropyl)trimethoxysilane (GOPTS) 2\% in toluene for $2 \mathrm{~h}$ at room temperature. After 2 h, the chips were washed with 2-propanol and air-dried. Next, the chips were baked for $30 \mathrm{~min}$ at $150{ }^{\circ} \mathrm{C}$ and after cooling at room temperature, they were immersed in a solution of propargylamine $2 \%$ in toluene for $4 \mathrm{~h}$. Finally, the chips were washed with 2-propanol, air-dried, and baked for $30 \mathrm{~min}$ at $150{ }^{\circ} \mathrm{C}$.

Probe immobilization studies. To perform this study, solutions of oligonucleotides T1.I, T2.I and T4.I at 2, 1 and $0.5 \mu \mathrm{M}$ were prepared in PBS $1 \times$ from a starting concentration of $20 \mu \mathrm{M}$ (50 $\mu \mathrm{l}$ of oligonucleotide $100 \mu \mathrm{M}, 150 \mu \mathrm{l}$ MilliQ water and $50 \mu \mathrm{l}$ of TCEP $0.1 \mathrm{M}$ in MilliQ water).

These solutions were spotted (40 nl/spot, humidity set at $95 \%$ ) onto the functionalized surfaces creating microarrays where each row contained 5 replicas (spots); the number of rows was nine (one row per oligo and concentration).

The microarrays were then exposed to UV-light at $365 \mathrm{~nm}$, with the lamp placed at a fixed distance $(5 \mathrm{~cm}$ ) from the slide, for $60 \mathrm{~min}$ to induce the immobilization (mono or multipoint attachment). Finally, slides were thoroughly rinsed with water and air-dried. By the SFR, fluorescence measurements let us to quantify the immobilization yield. Measurements were made by accumulation of emitted light by the samples during 15 seconds with a device gain of 3 .

Hybridization studies. Solutions of oligonucleotides T1.H, T2.H and T4.H 0.1, 0.2, 0.4, $0.51,2,3$ and $5 \mu \mathrm{M}$ were prepared in PBS $1 \times$ from a starting concentration of $20 \mu \mathrm{M}$. For each type of oligonucleotide, a microarray was printed on a functionalized surface (5 spots/row, $40 \mathrm{nl} /$ spot, 8 rows, humidity set at 95\%) using the robotic arrayer. The slides were then irradiated as before, rinsed with water, and air dried. Afterwards, $50 \mu \mathrm{l}$ of Target A $(0.5 \mu \mathrm{M}$ in SSC $1 \times)$ were spread over the entire surface with a coverslip. After incubation in a slim box for $45 \mathrm{~min}$ at $37^{\circ} \mathrm{C}$, the coverslip was gently removed and the chip washed with SSC $0.1 \times$ and air dried. The fluorescence intensity of the spots was registered with the SFR as described above.

Salmonella PCR products detection. Glass slides were cut in $2 \times 1 \mathrm{~cm}^{2}$ pieces and activated and functionalized with alkene groups as described above for silicon surfaces. Then microarrays of probes T1.Sal, T2.Sal, T4.Sal at $2 \mu \mathrm{M}$ in PBS1×, T1.I as immobilization control, and T4.Cam as non-specific hybridization control (both at $2 \mu \mathrm{M}$ ), were printed and immobilized as described before. 
1 After irradiation, washing and drying, the chips were ready for hybridization. Firstly, they were pre-hybridized in SSC $1 \times, 15 \%$ formamide, at $37^{\circ} \mathrm{C}$ for $30 \mathrm{~min}$. Then, $35 \mu \mathrm{l}$ of PCR product (dilutions ranging from $1 / 10$ to $1 / 100$ ) in SSC $1 \times, 15 \%$ formamide, were dispensed on the chips and spread out over the surface using a coverslip. The target PCR products were denaturalized at $95{ }^{\circ} \mathrm{C}$ for $5 \mathrm{~min}$ and then cooled down in ice for $2 \mathrm{~min}$ immediately before the hybridization. The chips were incubated at $37^{\circ} \mathrm{C}$ for $60 \mathrm{~min}$, then washed with SSC $0.1 \times$ and air dried.

For naked-eye detection, a mixture containing rabbit anti-digoxigenin antibody (1/10000) and gold labeled goat anti-rabbit antibody (1/100) in PBS-T were applied over the chip, and incubated for $30 \mathrm{~min}$ at room temperature. After washing with PBS-T, the chips were incubated with $20 \mu \mathrm{l}$ of silver developer solution, and after $12 \mathrm{~min}$, positive results (silver deposition) appeared on the microarrays.

For fluorescence detection and quantification, $30 \mu \mathrm{l}$ of anti-digoxigenin antibody produced in rabbit, 1/100 in PBS-T, were spread over the chip and incubated for $30 \mathrm{~min}$ at room temperature. After washing with PBS-T, $30 \mu \mathrm{l}$ of Alexa647-labeled goat antirabbit antibody, 1/50 in PBS-T, were incubated over the chip for another $30 \mathrm{~min}$ at room temperature. Finally, the chip was washed with PBS-T, water and air dried, and the fluorescence registered with the SFR.

DPI hybridization experiments. Unmodified Anachips were functionalized with alkenyl or alkynyl groups as described before. One of the channels was used to immobilize T1.H, while the other channel was employed to attach T2.H in one case, and T4.H in the other case. The spatial selectivity for the probe tethering only on one of the two channels available was achieved by selective irradiation using a homemade photomask. The chip was inserted in the device, and calibrated following fabricant instructions. The carrier buffer was SSC $1 \times$. Target B $5 \mu \mathrm{M}$ in SSC $1 \times$ was flowed over both channels for $25 \mathrm{~min}$ at a flow rate of $10 \mu \mathrm{l} / \mathrm{min}$. Afterwards, water ( $25 \mathrm{~min}, 10$ $\mu \mathrm{l} / \mathrm{min}$ ) was injected to dehybridize. Then, a non-complementary strand ( $25 \mu \mathrm{M}, 10$ $\mu \mathrm{l} / \mathrm{min}, 5 \mathrm{~min}$ ) was flowed to assess the specificity of the recognition.

\section{ASSOCIATED CONTENT}

\section{Supporting Information}

MALDI-TOF MS of oligonucleotides, Contact angle values, Calculated Immobilization densities, Calibration curve for Target A, AFM Surface characterization, XPS C1s peak 
1 deconvolution of alkene-ended biofunctionalized surfaces, Data obtained by DPI, Results

2 for a colorimetric microarray. This material is available free of charge via the Internet at

3 http://pubs.acs.org.

4

5 AUTHOR INFORMATION

6 Corresponding Author

7 *Phone: 34-963873415; fax: 34-963879349; E-mail: amaquieira@quim.upv.es

8 *Phone: 33-(0)-467144961; fax: 33-(0)-467042029; E-mail:

9 francois.morvan@umontpellier.fr

10

11

Notes

The authors declare no competing financial interest.

ACKNOWLEDGMENTS

The authors thank Dr. Elena Pinilla for her helpful discussion about AFM results. This work was funded by EU's program Horizon 2020 ICT-26-2014-644242, Spanish Ministry MINECO CTQ/2013/45875-R FEDER and local administration GVA PROMETEO II 2014/40. The authors acknowledge Tortajada-Genaro, Luis and Niñoles Rodenes, Regina for kindly providing the Salmonella and Campylobacter PCR products. F.M. is member of Inserm.

\section{Abbreviations}

GOPTS: (3-glycidyloxypropyl)trimethoxysilane, TCEP: tris(2-carboxyethyl)phosphine hydrochloride, THF: tetrahydrofurane, TCA: trichloroacetic acid, PBS: Phosphate buffer saline, SSC: sodium citrate saline buffer, SFR: surface fluorescence reader, DPI: Dual polarization , Interferometry, RSD: Relative standard desviation, , AFM: Atomic Force microscopy, XPS: X-ray photoelectron spectroscopy, TE: Transverse electrical, TM: Transverse magnetical, dsDNA: double strand DNA

\section{REFERENCES}

(1) Huys, I., Matthijs, G., and Van Overwalle, G. (2012) The fate and future of patents 
on human genes and genetic diagnostic methods. Nat. Rev. Genet. 13, 441-8.

(2) Sett, A., Das, S., and Bora, U. (2014) Functional nucleic-acid-based sensors for environmental monitoring. Appl. Biochem. Biotechnol. 174, 1073-91.

(3) Sett, A. (2012) Aptasensors in Health, Environment and Food Safety Monitoring. Open J. Appl. Biosens. 1, 9-19.

(4) Scheler, O., Glynn, B., and Kurg, A. (2014) Nucleic acid detection technologies and marker molecules in bacterial diagnostics. Expert Rev. Mol. Diagn. 14, 489-500.

(5) Sassolas, A., Leca-Bouvier, B. D., and Blum, L. J. (2008) DNA biosensors and microarrays. Chem. Rev. 108, 109-139.

(6) Ji, H., and Davis, R. W. (2006) Data quality in genomics and microarrays. Nat. Biotechnol. 24, 1112-1113.

(7) Rao, A. N., and Grainger, D. W. (2014) Biophysical properties of nucleic acids at surfaces relevant to microarray performance. Biomater. Sci. 2, 436.

(8) Heller, M. J. (2002) DNA Microarray Technology: Devices, Systems, and Applications. Annu. Rev. Biomed. Eng. 4, 129-153.

(9) Barbulovic-Nad, I., Lucente, M., Sun, Y., Zhang, M., Wheeler, A. R., and Bussmann, M. (2006) Bio-Microarray Fabrication Techniques-A Review. Crit. Rev. Biotechnol. 26, 237-259.

(10) Wu, P., Castner, D. G., and Grainger, D. W. (2008) Diagnostic devices as biomaterials: a review of nucleic acid and protein microarray surface performance issues. J. Biomater. Sci. Polym. Ed. 19, 725-53.

(11) Zhou, X. C., Huang, L. Q., and Li, S. F. Y. (2001) Microgravimetric DNA sensor based on quartz crystal microbalance: comparison of oligonucleotide immobilization methods and the application in genetic diagnosis. Biosens. Bioelectron. 16, 85-95.

(12) Pan, S., and Rothberg, L. (2005) Chemical Control of Electrode Functionalization for Detection of DNA Hybridization by Electrochemical Impedance Spectroscopy. Langmuir 21, 1022-1027.

(13) Manning, B., and Eritja, R. (2012) Functionalization of Surfaces with Synthetic 
1 Oligonucleotides,. Melba Navarro and Josep A. Planell (eds.), Nanotechnology in

2 Regenerative Medicine: Methods and Protocols, Methods in Molecular Biology, 811, 3 89-100.

4 (14) Singh, V., Zharnikov, M., Gulino, A., and Gupta, T. (2011) DNA immobilization, 5 delivery and cleavage on solid supports. J. Mater. Chem. 21, 10602.

6 (15) Nimse, S., Song, K., Sonawane, M., Sayyed, D., and Kim, T. (2014)

7 Immobilization Techniques for Microarray: Challenges and Applications. Sensors 14, $8 \quad 22208-22229$.

9 (16) Kolb, H. C., Finn, M. G., and Sharpless, K. B. (2001) Click chemistry Diveverse chemical funtion from a few good reactions. Angew. Chemie - Int. Ed. 40, 2004-2021.

(17) Hoyle, C. E., and Bowman, C. N. (2010) Thiol-ene click chemistry. Angew. Chemie - Int. Ed. 49, 1540-1573.

(18) Dondoni, A. (2008) The emergence of thiol-ene coupling as a click process for materials and bioorganic chemistry. Angew. Chemie - Int. Ed. 47, 8995-8997. (19) Massi, A., and Nanni, D. (2012) Thiol-yne coupling: revisiting old concepts as a breakthrough for up-to-date applications. Org. Biomol. Chem. 10, 3791. (20) Hoogenboom, R. (2010) Thiol-yne chemistry: A powerful tool for creating highly functional materials. Angew. Chemie - Int. Ed. 49, 3415-3417.

(21) Wendeln, C., Rinnen, S., Schulz, C., Arlinghaus, H. F., and Ravoo, B. J. (2010) Photochemical microcontact printing by thiol-ene and thiol-yne click chemistry. Langmuir 26, 15966-15971.

(22) Campos, M. a C., Paulusse, J. M. J., and Zuilhof, H. (2010) Functional monolayers on oxide-free silicon surfaces via thiol-ene click chemistry. Chem. Commun. 46, 55125514.

(23) Mehlich, J., and Ravoo, B. J. (2011) Click chemistry by microcontact printing on self-assembled monolayers: a structure-reactivity study by fluorescence microscopy. Org. Biomol. Chem. 9, 4108-4115.

(24) Bertin, A., and Schlaad, H. (2009) Mild and Versatile (Bio-)Functionalization of 
1 (25) Iwasaki, Y., and Ota, T. (2011) Efficient biotinylation of methacryloyl-

2 functionalized nonadherent cells for formation of cell microarrays. Chem. Commun.

3 (Camb). 47, 10329-10331.

4 (26) Wendeln, C., Rinnen, S., Schulz, C., Kaufmann, T., Arlinghaus, H. F., and Ravoo, 5 B. J. (2012) Rapid preparation of multifunctional surfaces for orthogonal ligation by 6 microcontact chemistry. Chem. - A Eur. J. 18, 5880-5888.

7 (27) Jonkheijm, P., Weinrich, D., Köhn, M., Engelkamp, H., Christianen, P. C. M., 8 Kuhlmann, J., Maan, J. C., Nüsse, D., Schroeder, H., Wacker, R., et al. (2008)

9 Photochemical surface patterning by the thiol-ene reaction. Angew. Chemie - Int. Ed. 47, 4421-4424.

(28) Weinrich, D., Köhn, M., Jonkheijm, P., Westerlind, U., Dehmelt, L., Engelkamp, H., Christianen, P. C. M., Kuhlmann, J., Maan, J. C., Nüsse, D., et al. (2010) Preparation of biomolecule microstructures and microarrays by thiol-ene photoimmobilization. ChemBioChem 11, 235-247.

(29) Weinrich, D., Lin, P. C., Jonkheijm, P., Nguyen, U. T. T., Schröder, H., Niemeyer, C. M., Alexandrov, K., Goody, R., and Waldmann, H. (2010) Oriented immobilization of farnesylated proteins by the thiol-ene reaction. Angew. Chemie - Int. Ed. 49, 12521257.

(30) Lin, P. C., Weinrich, D., and Waldmann, H. (2010) Protein biochips: Oriented surface immobilization of proteins. Macromol. Chem. Phys. 211, 136-144.

(31) Escorihuela, J., Bañuls, M. J., Puchades, R., and Maquieira, Á. (2012) DNA microarrays on silicon surfaces through thiol-ene chemistry. Chem. Commun. 48, 2116. (32) Escorihuela, J., Bañuls, M. J., Grijalvo, S., Eritja, R., Puchades, R., and Maquieira, Á. (2014) Direct covalent attachment of DNA microarrays by rapid thiol-ene “click” chemistry. Bioconjug. Chem. 25, 618-627.

(33) Escorihuela, J., Bañuls, M.-J., Puchades, R., and Maquieira, Á. (2014) Site-specific immobilization of DNA on silicon surfaces by using the thiol-yne reaction. J. Mater. Chem. B 2, 8510-8517. 
1 innovative and versatile polythiol probes for use on elosa or electrochemical biosensors:

2 Application in hepatitis c virus genotyping. Anal. Chem. 85, 9204-9212.

3 (35) Arnandis-Chover, T., Morais, S., Tortajada-Genaro, L. a., Puchades, R., Maquieira, 4 a., Berganza, J., and Olabarria, G. (2012) Detection of food-borne pathogens with DNA 5 arrays on disk. Talanta 101, 405-412.

6 (36) Santiago-Felipe, S., Tortajada-Genaro, L. A., Morais, S., Puchades, R., and

7 Maquieira, Á. (2015) Isothermal DNA amplification strategies for duplex

8 microorganism detection. Food Chem. 174, 509-515.

9 (37) Mira, D., Llorente, R., Morais, S., Puchades, R., Maquieira, A., and Marti, J.

10 (2004) High-throughput screening of surface-enhanced fluorescence on industrial

11 standard digital recording media, in European Symposium on Optics and Photonics for

12 Defence and Security (Carrano, J. C., and Zukauskas, A., Eds.), pp 364-373.

13 International Society for Optics and Photonics.

14 (38) Horcas, I., Fernández, R., Gómez-Rodríguez, J. M., Colchero, J., Gómez-Herrero, 15 J., Baro, A. M. (2007) WSXM: A Software for Scanning Probe Microscopy and a Tool 16 for Nanotechnology. Rev. Sci. Instrum.78, 013705-8. 
1 TOC

2 Controlling the solid-probe-fluid interface by probe multi-point attachment improves significantly 3 the hybridization process

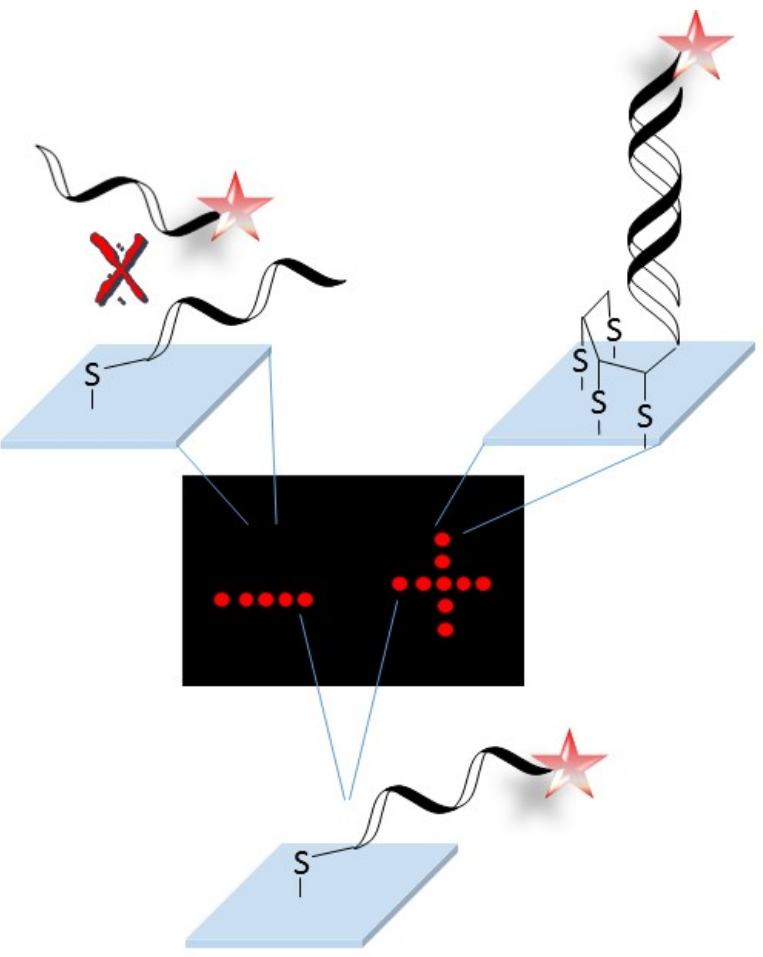

